\title{
Budesonide in Rhinological Practice
}

\author{
Ravi Ramalingam, N. Ahilasamy, K.K.R ENT Hospital and Research Institute \\ 827 Poonamallee High Road, Chennai - 600010
}

\section{|||||||||||||||||||||||||||||||||||||||||||||||||||||||||||||||||||||||||||||||||||||||||||||||||||||||||||||||| $\mid$}

$\mathbf{T}$ he management of Nasal Polyposis and $\mathrm{Al}$ lergic Rhinitis has under gone a significant improvement with the introduction of topical and systemic steroids. Topical glucocorticoids have been shown to be very effective in the prophylaxis and treatment of allergic rhinitis and nasal polyposis. These drugs have a high topical potency, with rapid systemic deactivation and so have minimal side effects. Budesonide is one of the most effective in this category of topical steroids drugs.

Budesonide is a non-halogenated corticosteroid and has shown a favorable relationship between anti-inflammatory effect and systemic effects due to the fact that Budesonide is inactivated rapidly in the liver after systemic absorption.

\section{Mechanism of action}

The action is mediated by the GCS receptors within the inflammatory and the immune cells. It stabilizes the mast cell membranes and makes the vascular endothelium less permeable. This ultimately results in

- Reduction of the plasma exudate

- Decreased edema formation

- Reduction in the mucosal thickening and hyper activity.

Inflammatory mediators like interleukins. granulocyte macrophage factor, natural killer cells etc are downgraded. It however, must be emphasized that topical steroids must reach the desired area to be effective. The preliminary use of a decongestant may improve the penetrance of the topical steroid preparation.

\section{Indications:}

- Prophylaxis and treatment of allergic rhinitis.

- Treatment of perennial rhinitis.

- Nasal polyposis

a) to induce the regression of small polyps

b) to reduce the recurrence rate after polypectorny/Endoscopic sinus Surgery

\section{Preparations}

a) Pressurized-metered dose inhaler or pressurized aerosol.

b) Water based suspension delivered from a metered dose pump spray - Aqua preparation

c) Inspiratory - flow driven metered dose inhaler.

\section{Dosage}

Buderoside (Rhinocort / Budenase nas.: spray) $200 \mathrm{mcg}$. Twice daily or $400 \mathrm{mcg}$. Single daily dosage form. 
In recurrent nasal polyposis $40 \mathrm{mcg}$ daily helps in preventing further recurrence following surgical intervention. It has to be continued for a minimum period of 3-6 months. Most of the patients usually get a subjective reduction in nasal symptoms with improvement of nasal blockade, smell perception and also improved sinus ventilation.

In cases of seasonal and perennial allergic rhinitis, Budesonide is found to be most appropriate in getting relief from nasal symptoms

\section{Side Effects}

\section{Local Effects}

Budesonide is usually well tolerated. Some of the side effects include.

- Nasal dryness

- Irritation

- Epistaxis

- Crusting
All are mild and transient.

There have been reports of ulceration of nasal mucous membrane and septal perforation

Systemic effects are significantly reduced because of its rapid and efficient metabolic inactivation to low metabolites.

Studies on animals have shown to cause abnormalities of fetal development, so caution is advisable in pregnant patients.

In conclusion topical glucocorticosteroids especially Budesonide has advantages of

1) Non sedation unlike traditional antihistamines

2) No association with Rhinitis medicamentosa

3) Effectiveness against all the symptoms of rhinitis like rhinorrhoea, nasal itching, sneezing and nasal congestion. 